\title{
Self-restoring polymer brushes under tribological stress and the biomedical applications
}

Røn, Troels; Javakhishvili, Irakli; Hvilsted, Søren; Jankova, Katja; Lee, Seunghwan

Published in:

MRS Advances

Link to article, DOI:

10.1557/adv.2016.345

Publication date:

2016

Document Version

Peer reviewed version

Link back to DTU Orbit

Citation (APA):

Røn, T., Javakhishvili, I., Hvilsted, S., Jankova, K., \& Lee, S. (2016). Self-restoring polymer brushes under tribological stress and the biomedical applications. MRS Advances, 1(27), 1971-1976.

https://doi.org/10.1557/adv.2016.345

\section{General rights}

Copyright and moral rights for the publications made accessible in the public portal are retained by the authors and/or other copyright owners and it is a condition of accessing publications that users recognise and abide by the legal requirements associated with these rights.

- Users may download and print one copy of any publication from the public portal for the purpose of private study or research.

- You may not further distribute the material or use it for any profit-making activity or commercial gain

- You may freely distribute the URL identifying the publication in the public portal

If you believe that this document breaches copyright please contact us providing details, and we will remove access to the work immediately and investigate your claim. 


\title{
Self-restoring polymer brushes under tribological stress and the biomedical applications
}

\author{
Troels Røn, ${ }^{1}$ Irakli Javakhishvili, ${ }^{2}$ Søren Hvilsted, ${ }^{2}$ Katja Jankova, ${ }^{2}$ Seunghwan Lee ${ }^{1}$ \\ ${ }^{1}$ Department of Mechanical Engineering, Technical University of Denmark, DK-2800 Kgs. \\ Lyngby, Denmark \\ ${ }^{2}$ Danish Polymer Centre, Department of Chemical and Biochemical Engineering, Technical \\ University of Denmark, DK-2800 Kgs. Lyngby, Denmark
}

\begin{abstract}
For biological and mechanical systems involving moving parts, surface slipperiness is often a critical attribute for their optimal functions. Surface grafting with hydrophilic polymers is a powerful means to render materials slippery in aqueous environment. In "inverted grafting-to approach", the hydrophilic polymer chains of amphiphilic diblock copolymers dispersed within a poly(dimethylsiloxane) (PDMS) network are selectively segregated upon exposure to aqueous solution. This allows formation of extremely stable brush-like polymer layers. Tribological application of inverted grafting-to approach was successfully demonstrated with PDMS-blockpoly(acrylic acid) (PDMS- $b$-PAA) dispersed within thin PDMS films on PDMS blocks by showing friction coefficients $(\mu)$ of ca $10^{-2}$ to $10^{-3}$, depending on the load, $\mathrm{pH}$ and buffer salinity in the absence of other external re-supply of PAA chains. Further manipulations of the thin PDMS film incorporating PDMS- $b$-PAA to optimize the tribological properties are presented. Lastly, first trials to employ PAA-grafted PDMS surface to generate in-vitro mucosae model are also presented and discussed.
\end{abstract}

\section{INTRODUCTION}

Surface grafting of materials in good or theta solvents have shown a broad range of applications, including colloidal stabilization, antifouling, and lubrication [1]. These properties are realized via the formation of highly solvated and stretched "brush-like" polymer chains on surfaces.

Improved slipperiness of hydrophobic materials in aqueous environment by hydrophilic brush layer is an excellent example of exploiting synergy between polymer brush and solvent as the repulsion of the brush-like polymer layers against wall or opposing brush layer, together with the formation of fluid-like layer between gliding surfaces, allows for water to be employed as base stock [2]. Two most widely employed methods to date to graft polymer chains, particularly for aqueous lubrication purposes, are "grafting-to" and "grafting-from" approaches. For the former, pre-made polymer chains are dissolved in base stock (aqueous solution) and polymers are spontaneously attracted to the surface driven by physisorption. Thus, it is convenient for final application, but the polymer films are too easily rubbed away by the tribological stress unless excess polymers are present in bulk solution to restore the lubricating films. Grafting-to approach, in which polymerization occurs from the surface, can be a solution for stronger anchoring of polymer chains. Nevertheless, surface polymerization process is typically cumbersome and costly. More importantly, once the lubricating polymer layer is damaged by the tribological stress, it cannot be revived until polymer formation process is restarted from the beginning. Recently, we have shown that another approach to graft polymers can be optimally utilized for tribological application [3] (Figure 1). 


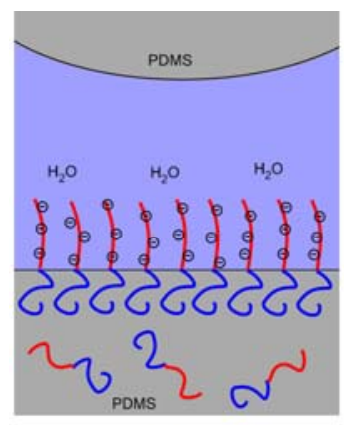

(a)

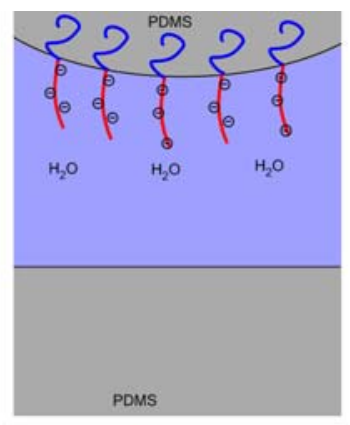

(b)

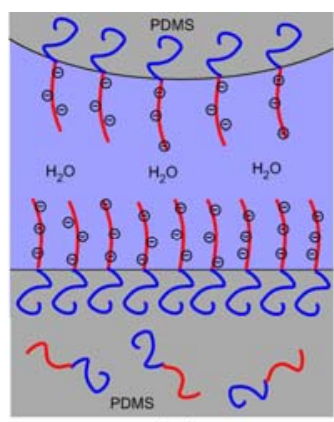

(c)

Figure 1. A schematic illustration of inverted grafting-to approach to graft hydrophilic polymer chains on PDMS surface: (a) thin PDMS film incorporating PDMS- $b$-PAA diblock copolymers are coated on (a) PDMS substrate only (b) PDMS pin only (c) both PDMS pin and disk.

In this approach, amphiphilic diblock copolymers, e.g. poly(dimethylsiloxane)-blockpoly(acrylic acid) (PDMS- $b$-PAA), are mixed with castable PDMS so that they can be dispersed within PDMS network after curing. This PDMS thin film incorporating diblock copolymers can be deposited on various materials' surfaces. Upon exposure to water [4], or often after applying initiating tribostress in water, hydrophilic blocks (PAA) of the copolymers are selectively segregated from the PDMS network to form brush-like layers, whereas the hydrophobic blocks (PDMS) remain trapped inside the PDMS network acting as anchors for the hydrophilic polymer brushes. Excellent anchoring stability of hydrophilic brush layer is a distinct novelty of this system, indebted from high reluctance of hydrophobic blocks to get exposed to aqueous solution and/or from facile replenishment of the diblock copolymers in case of film damage. Thus, a long-term lubricating performance was readily achieved [3]. Moreover, facile grafting of hydrophilic polymer chains on compliant material surfaces, e.g. silicone rubber, demonstrates high potential of this approach in biomedical applications related to catheter, contact lens, syringe sealant, and valves. In the present study, we have examined the influence of location of PDMS thin film layers between two surfaces in relative motion. In turn, we have also examined the effect of mixing PDMS- $b$-PAA with poly(dimethylsiloxane)-block-poly(ethylene glycol) (PDMS- $b$-PEG) on the formation of hydrophilic polymer chains. Lastly, a first biomedical application to generate in vitro mucosae model was also tested.

\section{EXPERIMENTAL DETAILS}

PDMS samples were prepared with a Sylgard® 184 kit (Dow Corning, Midland, MI) according to a standard procedure $[5,6]$. The diameter/thickness of disk was $30 / 5 \mathrm{~mm}$ and the radius of pin was $3 \mathrm{~mm}$. Additional PDMS thin film can be added by spin-coating when nanometer scale film thickness is targeted [3]. In the present study, this film was prepared by dripping ca. 10 drops of a THF/ $n$-heptane $(1: 4, \mathrm{vol} / \mathrm{vol})$ solution containing the mixture of amphiphilic diblock copolymer PDMS- $b$-PAA or PDMS- $b$-PEG and uncrosslinked PDMS, leading to 3-4 $\mu \mathrm{m}$ thickness. The ratio for diblock copolymer and PDMS mix was 1:4 (wt/wt) and the total polymer in the cosolvent (THF/n-heptane) was $5 \mathrm{wt} \%$. Details on synthesis and analyses of the diblock copolymers were addressed elsewhere [3]. After dripping, the solvent evaporated relatively fast. PDMS pins and disks were then cured at $70{ }^{\circ} \mathrm{C}$ overnight again. The samples were abbreviated 
as "DC20\%-PDMS(4.7)- $b$-PAA(6.1)" and "DC20\%-PDMS(4.7)-b-PEG(2.1)" in order to denote the coating method ("DC" for drip coating), wt $\%$ of the diblock copolymer ("20\%"), and the molecular weights of hydrophobic PDMS and hydrophilic PAA or PEG blocks (the numbers in brackets in $\mathrm{kDa}$ ). The lubricating capabilities of the drip-coated films in an aqueous environment were assessed by means of pin-on-disk tribometry (CSM Instruments with 4.4 M software version). Tribological measurements were conducted in HEPES buffer solutions (1 mM HEPES) with varying $\mathrm{pH}$ and additional $\mathrm{NaCl}$ concentration or phosphate-buffered saline (PBS). The sliding speed was varied from 100 to $0.25 \mathrm{~mm} \cdot \mathrm{s}^{-1}$. The load was controlled by applying dead weight on the pin (either 1 or $2 \mathrm{~N}$ ). The friction coefficient $(\mu)$ was calculated from the following equation: $\mu=F_{\text {friction }} / F_{\text {load. }}$ For in vitro mucosae model experiments, egg white was obtained from chicken eggs from local supermarket and porcine gastric mucin (PGM) was purchased from Sigma Aldrich (Type III; partially purified). Tribopairs were exposed to PGM solution overnight before commencement of friction measurements.

\section{DISCUSSION}

Figure 2 shows the $\mu$ vs speed plots obtained from the sliding contacts of PDMS-PDMS with varying surface treatments of pins and disks with the PDMS thin films and different aqueous solution conditions $($ load $=2 \mathrm{~N})$.

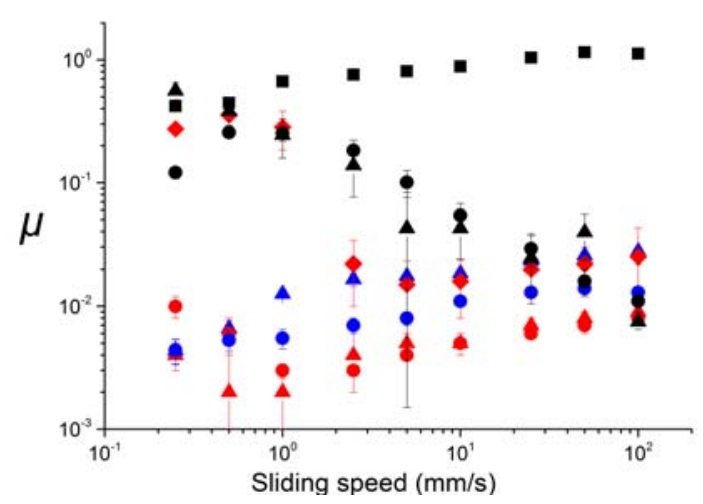

Figure 2. $\mu$ vs. speed plots obtained from PDMS-PDMS sliding contacts with different locations of the DC20\%PDMS(4.7)-b-PAA(6.1) coating and solution conditions (HEPES, $1 \mathrm{mM}$ ). $\mathbf{\square}$ : no coating, $\mathrm{pH} 7.0, \boldsymbol{\Delta}$ : disk only, $\mathrm{pH} 7.0, \boldsymbol{\Delta}$ : disk only, $\mathrm{pH} 7.0+150 \mathrm{mM} \mathrm{NaCl}, \boldsymbol{\Delta}$ : disk only, $\mathrm{pH}$ 9.0, : pin only, $\mathrm{pH} 9.0$, $\mathbf{0}$ : both pin and disk, $\mathrm{pH} 7.0$, $\mathrm{O}$ : both pin and disk, $\mathrm{pH} 7.0+150 \mathrm{mM}$ $\mathrm{NaCl}$, both pin and disk, $\mathrm{pH} 9.0$. Applied load was $2 \mathrm{~N}$.

The lubricating efficacy of PAA-grafted PDMS surfaces via inverted grafting-to approach was highly dependent on aqueous solvent conditions. For PAA brush film grafted on PDMS disk only (Figure 1(a)), $\mu$ values of $10^{-3}-10^{-2}$ were observed over the entire speed range at $\mathrm{pH} 9.0$. This represents more than two orders of magnitude decrease in $\mu$ compared to sliding contacts of bare PDMS-PDMS tribopair under the same condition. The $\mu$ values of ca. $10^{-2}$ or lower were also observed at $\mathrm{pH} 8.0$ and even $\mathrm{pH} 7.5$ (data not shown). But, at $\mathrm{pH} 7.0$, the $\mu$ values showed a gradual increase from ca. $10^{-2}$ to $5 \times 10^{-1}$ with decreasing speed (Figure 2 ). The dependence of the lubricity of segregated PAA chains on $\mathrm{pH}$ is related to the degree of ionization $(\alpha)$ $\mathrm{COOH} / \mathrm{COO}^{-}$in PAA. Estimated free energy change per area from the hydration of fully 
protonated PAA $(\alpha=0)$ is only $-214 k_{B} T / \mathrm{nm}^{2}$ and is substantially smaller than that of -1365 $k_{B} T / \mathrm{nm}^{2}$ for fully deprotonated PAA $(\alpha=1.0)$ [3]. This difference is ascribed to stronger hydration of $\mathrm{PAA}^{-}$than PAA. The degree of ionization of PAA(6.1) was estimated to be 0.61 , 0.94 and 0.99 at $\mathrm{pH} 7.0,8.0$, and 9.0, respectively [3]. Meanwhile, addition of $150 \mathrm{mM} \mathrm{NaCl}$ decreased the $\mu$ values at $\mathrm{pH} 7.0$ to a level at $\mathrm{pH} 8.0$ again, which can be rationalized by the screening of electrostatic repulsion between $\mathrm{PAA}^{-}$chains at $\mathrm{pH}$ 7.0, thus increasing $\alpha$ value. However, as salts have secondary effect of reducing the gyration of charged polymer chains by screening the electrostatic repulsions [7], the lubricity at $\mathrm{pH} 7.0$ with $150 \mathrm{mM} \mathrm{NaCl}$ was not as good as that at pH 9.0 with no salt (Figure 2).

The lubricating efficacy was also dependent on the locations of PAA chains at the tribological interface. Drip-coating of PDMS pin alone was observed not as effective in the lubricity as drip-coating of PDMS disk alone (Figure 1(b)). In fact, this is a general trend for unsymmetrical tribopairs [8]. As pin in tribometer functions as slider, it is under a tribological stress, whereas disk experiences tribostress only periodically. It was of interest whether the lubricating properties of the system can be further improved when both PDMS pin and disk were drip-coated with PDMS thin film (Figure 1(c)) as brush-brush repulsion may additionally contribute to improve lubricity [2]. Some magnitude of further reduction in $\mu$ was observed at pH 7.0 with $150 \mathrm{mM} \mathrm{NaCl}$. However, at pH 9.0, nearly the same results as PDMS disk only coating was observed. This could be related to the detection limit of the tribometer. But, it could also originate from that the drip coating on PDMS pin led to some increase in surface roughness.

A unique feature of PDMS thin film is that a hydrophobic "skin layer" is generated on the very top of the surface and impedes spontaneous segregation of hydrophilic PAA chains [3]. Skin layer may arise from the arrangement of PDMS- $b$-PAA diblock copolymer exposing hydrophobic PDMS blocks to ambient and/or migration of uncrosslinked silicone moieties to the surface. The presence of skin layer was manifested by an initial high friction ( $\mu$ reaching as high as $3 \times 10^{-1}$ ) upon exposure to tribological stress at the first few laps, although it is rapidly reduced $\left(\mu<10^{-2}\right)$, indicating removal of the skin layer. To explore a possibility to suppress the skin layer effect, PDMS(4.7)- $b$-PAA(6.1) was mixed with PDMS(4.7)- $b$-PEG(2.1) (4:1 wt. ratio, in total of $20 \mathrm{wt} \%$ in the film). This is based on the idea that PDMS(4.7)- $b$-PEG(2.1) is smaller in size (radius of gyration) and more readily segregates without the need of initial tribostress due to the smaller surface repulsion of the lower polarity PEG compared to PAA [3]. As shown in Figure 3, the initial high friction forces were much more suppressed; $\mu$ values were only up to 2 $\times 10^{-2}$ at first few laps.

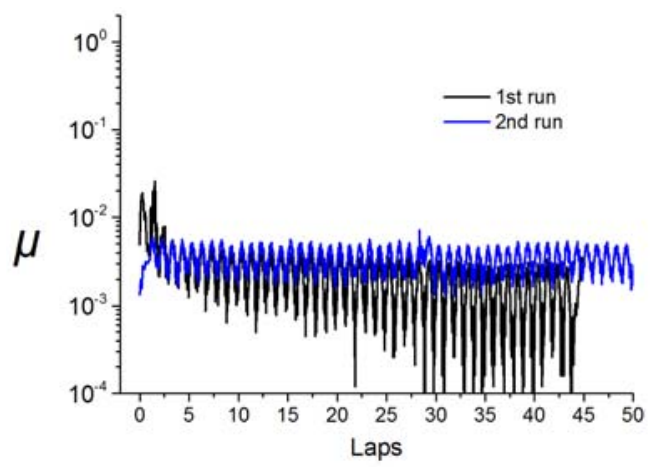

Figure 3. $\mu$ vs laps for the PDMS thin films incorporating the diblock copolymers of PDMS(4.7)- $b$-PAA(6.1) and PDMS(4.7)- $b$-PEG(2.1) (80\%:20\%, for the total diblock copolymers) in HEPES (1 mM, pH $7.0+150 \mathrm{mM} \mathrm{NaCl})$ : speed, $0.5 \mathrm{~mm} / \mathrm{s}, 1 \mathrm{~N}$ load. 


\section{The first biomedical application}

Given that PAA-brushes can be readily generated on compliant PDMS substrate via inverted grafting-to approach, it was exploited to generate in vitro mucosae model. In vitro mucin/mucus layers model is useful for a variety of scientific research where the interaction of mucus with foreign bodies is important, such as drug delivery or entrapment of bacteria. A focused interest of this study is to provide in vitro mucosae model with which mechanical or tribological tests can be performed. Slipperiness of tissue-contacting biomedical devices, such as endoscope, catheter, or contact lenses, is important for optimal operation and minimal damage to the tissue. In vitro mucosae model is expected to help assess and optimize surface slipperiness of those biomedical devices. PAA is a well-known mucoadhesive polymer the layer of which is expected to interact and generate mucus/mucin layers. In this study, we have chosen egg white and porcine gastric mucin (PGM) as model mucus and mucin molecules, respectively. After spontaneous adsorption of either egg white or PGM molecules onto PAA-grafted PDMS surface, sliding contacts were applied against bare PDMS pin by means of pin-on-disk tribometry. SC-20\% PDMS(4.7)- $b$ PAA(6.1) mentioned above was employed. The results are shown in Figure 4.
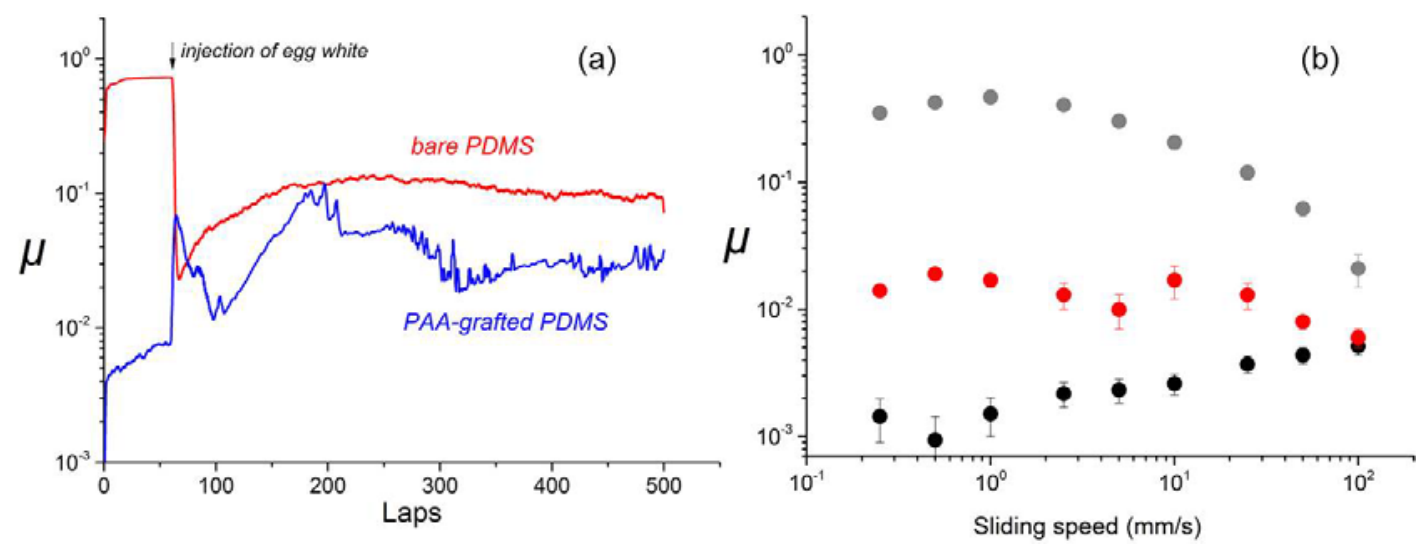

Figure 4. (a) $\mu$ vs laps between bare PDMS pin against either bare PDMS (red lines) or PAAgrafted PDMS (blue lines) in the presence of egg white $(2 \mathrm{~mL})$ in PBS $(2 \mathrm{~mL})(2.5 \mathrm{~mm} / \mathrm{s}$ and 2 N). (b) $\mu$ vs speed plots obtained from the sliding contact of PDMS/PDMS in PBS with PGM solution (10 mg/mL PGM) (๑), PDMS/PAA-PDMS in PBS pH 7.4(๑), and PDMS/PAAPDMS in PBS with PGM solution (10 mg/mL PGM) (๑), pH 7.4.

In Figure 4(a), egg white ( $2 \mathrm{~mL}$ ) was injected into a tribocup where bare PDMS pin is sliding in either bare PDMS or PAA-grafted PDMS in PBS (under $2 \mathrm{~N}$ load). The friction force between PDMS pin and disk was initially very high $\left(\mathrm{ca} .7 \times 10^{-1}\right)$. When egg white was injected into PBS, the friction forces dropped instantaneously as low as ca. $2 \times 10^{-2}$, but gradually increased to ca. 1 $\times 10^{-1}$ again. The same experiment was repeated with PAA-grafted PDMS substrate. As expected, the initial $\mu$ values were very small $\left(<10^{-2}\right)$ due to excellent lubricating properties of grafted PAA chains as shown above. Upon injection of egg white, a sudden increase of $\mu$ was observed, followed by a fluctuation between $10^{-2}$ and $10^{-1}$. Higher $\mu$ values compared to PAAgrafted PDMS substrate implies that a strong interaction occurred between egg white and the surface. It appears though this interaction is very complex and further investigation is necessary to understand the detailed interaction mechanism, especially under tribological stress. PGM 
solution $(10 \mathrm{mg} / \mathrm{mL}$ in PBS) was also employed in order to generate mucin layers on either bare or PAA-grafted PDMS surface (Figure 4(b)). Friction measurements were performed after overnight exposure of the PDMS surfaces to the PGM solution. PGM layers formed on PAAPDMS surfaces again showed an intermediate $\mu$ values between those formed on bare PDMS and bare PAA-PDMS surfaces at neutral $\mathrm{pH}$, which also support the interaction between PGM and PAA layers. Future studies should clarify the interaction of mucin/mucus with PAA-PDMS surfaces before and after tribological tests. Optimization of mucin/mucus layers to provide stable frictional properties is essential for reliable preclinical tests of biomedical tube-style devices.

\section{CONCLUSIONS}

In this study, we have demonstrated that PDMS surfaces grafted with PAA chains via inverted grafting-to approach display extremely low friction coefficient without supply of PAA from external sources. In this approach, a thin PDMS film incorporating PDMS- $b$-PAA is drip-coated on tribopair surfaces (e.g. PDMS rubber), and cured for crosslinking. Upon exposure to aqueous solution $(\mathrm{pH} \geq 7.5$ or $\mathrm{pH} 7$ with additional $150 \mathrm{mM} \mathrm{NaCl}$ ), or often by applying initiating tribostress, hydrophilic PAA chains are selectively segregated from the thin PDMS film to generate charged, hydrophilic, and slippery brush layers. Initial high friction forces, originating from the formation of ultrathin, hydrophobic skin layer in hydrophobic ambient environment, were significantly suppressed by incorporating smaller sized PDMS- $b$-PEG simultaneously. Coating of PDMS pin alone provided less effective lubricity than coating of PDMS disk alone due to more persistently applied tribostress on the slider. Exceptionally stable PAA layers generated on compliant PDMS surfaces via inverted grafting-to approach were employed to generate in vitro mucosae model by exposing to either egg white or PGM solutions. Tribological tests have shown a clear indication of the interaction between egg white/PGM with PAA-grafted PDMS surfaces. But, highly fluctuating friction forces should be further optimized for more reliable preclinical tests for biomedical devices to be applied in contacts with mucosae.

\section{ACKNOWLEDGMENTS}

The financial support from the Danish Council for Independent Research (DFF-FTP, 10-082707) and European Research Council (ERC Starting Grant 2010, 261152) are greatly appreciated.

\section{REFERENCES}

1. R. C. Advincula, W. J. Brittain, K. C. Caster and J. Ruhe, Polymer brushes: Synthesis, characterization, applications, (Wiley-VCH Verlag GmbH \& Co. KGaA, 2004).

2. J. Klein, Friction 1, 1 (2013).

3. T. Røn, I. Javakhishvili, S. Hvilsted, K. Jankova and S. Lee, Adv. Mater. Interf. 3, 1500472 (2016).

4. M. Inutsuka, N. L. Yamada, K. Ito and H. Yokoyama, ACS Macro Lett. 2, 265 (2013).

5. K. Chawla, S. Lee, B. P. Lee, J. L. Dalsin, P. B. Messersmith and N. D. Spencer, J. Biomed. Mater. Res. A 90, 742 (2008).

6. R. Heeb, R. M. Bielecki, S. Lee and N. D. Spencer, Macromolecules 42, 9124 (2009).

7. B. Lego, W. G. Skene and S. Giasson, Macromolecules 43, 4384 (2010).

8. S. Lee, M. Heuberger, P. Rousset and N. D. Spencer, Tribol. Lett. 16, 239 (2004). 